EPJ Web of Conferences 97,00018 (2015)

DOI: 10.1051/epjconf/201597000 18

(C) Owned by the authors, published by EDP Sciences, 2015

\title{
Exotic resonance production from heavy ion collisions
}

\author{
Su Houng Lee ${ }^{1, a}$ and Sungtae $\mathrm{Cho}^{2, \mathrm{~b}}$ \\ ${ }^{1}$ Department of Physics and Institute of Physics and Applied Physics, Yonsei University, Seoul 120-749, \\ Korea \\ ${ }^{2}$ Division of Science Education, Kangwon National University, Chuncheon 200-701, Korea
}

\begin{abstract}
In this talk, I will first summarize the recent interests in heavy multiquark configurations and then discuss the production of light nuclei from heavy ion collisions and what it tells us about the production of molecular configuration in these collisions. Next, I will discuss the intrinsic differences between a compact multiquark state and a normal hadron or a hadronic molecule. Finally, using the coalescence model, I will then show that the intrinsic differences lead to appreciable differences in the yields of these particles from a heavy ion collision depending on their assumed internal structure.
\end{abstract}

\section{Recent findings of exotic candidates}

We have seen a renewal of hadronic physics in recent years, which was triggered by the discovery of the $D_{s J}(2317)$ and $X(3872)$ states by the BaBar collaboration [1] and the Belle collaboration [2] respectively. Because the observed masses did not fit well within the quark model predictions, the states were speculated to be of tetra-quark or molecular configurations [3]. Furthermore, both the $X(3872)$ [4] and the charged charmonium state $Z(4430)$ [5] were recently confirmed at LHC.

Multiquark configurations were predicted long ago in the light quark sector [6,7]. But even to this date, there is no definite proof that the scalar nonets are crypto-exotic states and no exotic multiquark states have been found. In a series of papers $[8,9]$, together with the ExHIC collaboration, we have shown that the yields of a hadron can be used to discriminate its structure between a multiquark configuration and a normal or molecular configuration. In this talk, I will present few arguments justifying the claim.

\section{Random comments using the bag model}

\subsection{Quark density}

A critical difference between a multiquark configuration and a normal hadron or molecular configuration composed of usual hadrons is the density of quarks within the hadron. In a compact multiquark configuration, the quarks are confined within a region not so much different from the size of a normal hadron. This is true in most cases with central potential, as long as different quantum numbers allow

\footnotetext{
a e-mail: suhoung@yonsei.ac.kr

be-mail: sungtae.cho@kangwon.ac.kr
}

This is an Open Access article distributed under the terms of the Creative Commons Attribution License 4.0, which permits unrestricted use, distribution, and reproduction in any medium, provided the original work is properly cited. 


\begin{tabular}{|c|c|c|c|}
\hline & $\begin{array}{c}\text { Normal } \\
\text { meson }\end{array}$ & Tetraquark & Molecule \\
\hline & & & (d) \\
$\begin{array}{c}\text { Geometrical } \\
\text { configuration }\end{array}$ & dd & da dut & \\
\hline $\begin{array}{c}\text { Flavor } \\
\text { quantum } \\
\text { number }\end{array}$ & ud & ud & ud \\
\hline
\end{tabular}

Figure 1. Pictorial difference between a normal hadron, a tetraquark and a molecule.

them to occupy the lowest s-wave states. This is also true in a simple bag model. To see this, consider $N_{q}$ quarks confined within a bag of radius $R$ and bag pressure $B$. The nucleon energy is given as

$$
E_{\text {nucleon }}=N_{q} \frac{\omega}{R}+B \frac{4 \pi}{3} R^{3},
$$

where $\omega$ is just a constant representing the first mode. Typically, the radius can be obtained from $d E_{\text {nucleon }} / d R=0$ from which we obtain

$$
R^{4}=\frac{N_{q} \omega}{4 \pi B}
$$

The density of quarks inside the bag is then given as

$$
\rho_{\text {quark }}=\frac{N_{q}}{V}=\frac{N_{q}}{\frac{4 \pi}{3} R^{3}} \propto N_{q}^{1 / 4},
$$

that is to say, the density of quark increases with the number of quarks inside the bag. Therefore, the naive picture presented in Fig. 1, which shows a more heavily occupied multiquark state compared to its normal hadronic or molecular counterpart seems true.

\subsection{Vacuum inside the bag}

The bag constant $B$ is related to the difference between the gluon condensate inside and outside the bag. Using the nucleon mass in the chiral limit and the energy momentum tensor, one can estimate the gluon condensate in the nuclear matter. Extrapolating the formula to 5 times the nuclear matter density, one finds that the gluon condensate decreases to $70 \%$ of its vacuum value [10]. This suggests 
that if the bag occupies $1 / 5$ of the total volume in nuclear matter, the gluon condensate inside the bag would be $70 \%$ of its vacuum value. One then finds that the bag pressure is $B=\frac{1}{4}\left(-\frac{9}{8} \Delta\left\langle\frac{\alpha}{\pi} G^{2}\right\rangle\right) \approx$ $(190 \mathrm{MeV})^{4}$, consistent with previous estimates. But what is more striking is the similarity of the decrease of the gluon condensate seen at the critical temperature $T_{c}$ from lattice calculation, which also shows a $70 \%$ decrease from its vacuum value $[10,11]$. This suggests that the vacuum at $T_{c}$ is similar to that inside the bag. This is somehow in line with the "clearing vacuum picture" introduced in reference [12].

\section{Particle production in heavy ion collisions}

It is well known that hadron production in elementary processes follows factorization theorem combining the structure function, fragmentation function and elementary QCD cross section. On the other hand, over the years, it has been noted that ground state hadron production in heavy ion collisions follows statistical model prediction and that the chemical freezeout points from AGS, SPS and RHIC follow a line of constant energy per particle or an almost constant baryon and antibaryon density [13]. Moreover, coalescence model was able to explain intriguing features such as the enhanced production of baryons at midrapidity in the intermediate transverse momentum region and the quark number scaling of the elliptic flow of identified hadrons [14, 15].

Considering the equivalence/similarities between statistical model and coalescence model, one can picture the following hadronization process. Namely, at the initial thermalized temperature, quarkgluon plasma state is formed; as the system cools down to the critical point, the density of quarks and the vacuum changes to a state similar to that of inside the bag; at that point hadronization takes place statistically through coalescence, that is overlap of the configuration into the final hadron wave functions. The important feature, especially for the multiquark states or the excited states, is the overlap integral. The overlap integral would be small if the density of final hadrons are either smaller or larger than that at the hadronization point. Such additional wave function effect was used to explain the suppressed production of $\Lambda *$ over its statistical model prediction $[16,17]$.

\subsection{Production of light nuclei}

The production of light nuclei from heavy ion collisions is also found to follow statistical model prediction with parameters fitted to reproduce the usual hadron yield[18]. This is a surprise considering the fact that the formation of light nuclei will take place at the later stages of the collision where the nucleons decouple from the hadron gas near the kinetic freezeout temperature.

Let us first discuss what this experimental fact implies. Experimentally, the yield of the nucleon and deuteron and light nuclei follows statistical model, which in the nonrelativistic limit limplies

$$
\begin{aligned}
N_{p}^{\text {stat }} & =\gamma_{p} g_{p} V_{H}\left(\frac{m_{p} T_{H}}{2 \pi}\right)^{3 / 2} e^{-m_{p} / T_{H}}, \\
N_{D}^{\text {stat }} & =\gamma_{p}^{2} g_{D} V_{H}\left(\frac{m_{D} T_{H}}{2 \pi}\right)^{3 / 2} e^{-m_{D} / T_{H}} .
\end{aligned}
$$

Here, $\gamma, g, m$ are the fugacity, degeneracy and mass of nucleon or deuteron depending on the subscript. $V_{H}, T_{H}$ are the volume at the central rapidity region and temperature at the hadronization point, which we take to be the chemical freezeout parameters. It should be noted that the final number of the nucleons should include the contributions from excited states. These contribution will increase the number of nucleon as predicted by statistical model by a factor of two or so. We will come back to 
Table 1. Comparison of phase space at chemical freezeout time and nucleon coalescence time

\begin{tabular}{llll}
\hline$T$ & Volume & Phase space & (Phase space)/Volume \\
\hline$T_{H}=175 \mathrm{MeV}$ & $V_{H}=1908 \mathrm{fm}^{3}$ & $1 /\left(\frac{m_{p} T_{H}}{4 \pi}\right)^{3 / 2}=5.1 \mathrm{fm}^{3}$ & 0.0027 \\
$T_{F}=125 \mathrm{MeV}$ & $V_{F}=11322 \mathrm{fm}^{3}$ & $\frac{\left(4 \pi \sigma_{i}^{2}\right)^{3 / 2}}{1+2 \mu_{i} T_{F} \sigma^{2}}=29 \mathrm{fm}^{3}$ & 0.0026 \\
\hline
\end{tabular}

the issue later as it does not change the general argument that we will be discussing here. One can rewrite the deuteron number as follows.

$$
N_{D}^{\text {stat }}=\frac{g_{D}}{g_{p}^{2}} N_{p}^{\text {stat }}\left(N_{p}^{\text {stat }} \frac{1 /\left(\frac{m_{p} T_{H}}{4 \pi}\right)^{3 / 2}}{V_{H}}\right)
$$

Here, we have assumed that the mass of the deuteron is twice the mass of the nucleon. The factor in the big bracket is a typical multiplicative factor that multiplies whenever there is an extra nucleon. So for example, a similar factor would be additionally multiplied for the production rate of the triton.

While the number of the deuteron produced follows that given in Eq.(5), physics wise, it will form at a much later stage through the coalescence of the nucleons. Using the coalescence formula in the nonrelativistic limit with a gaussian form for the wave function of the deuteron, one finds

$$
N_{D}^{\text {Coal }}=\frac{g_{D}}{g_{N}^{2}} N_{N}\left(\frac{N_{N}}{V_{F}} \frac{\left(4 \pi \sigma^{2}\right)^{3 / 2}}{1+2 m_{p} T_{F} \sigma^{2}}\right) .
$$

The reason why the coalescence formula at very low temperature is similar to that of the statistical prediction is because the phase space occupied by a nucleon is similar at later time. That is the magnitude of the big bracket in Eq.(5) similar to that in Eq.(6) as can be seen in Table 1; a result consistent with the conservation of entropy carried by a single nucleon in a heavy ion collision [19].

The observation of the triton or helium also follows statistical model. That implies that the formulas obtained by further multiplying the large brackets in Eq.(5) and Eq.(6), depending on the number of nucleons, are valid. On the other hand, one should note that for the deuteron we have used $\sigma=4$ $\mathrm{fm}$ to account for the large size of the deuteron as for simple harmonic oscillator $\left\langle r^{2}\right\rangle=\sigma^{2} / 4$, the value of $\sigma$ should be smaller for helium or triton. This suggest that the formation of these light nuclei will take place earlier where the total volume is smaller such that

$$
\left.\frac{\left(4 \pi \sigma_{i}^{2}\right)^{3 / 2}}{1+2 \mu_{i} T \sigma_{i}^{2}}\right|_{\text {deuteron }}=\left.\frac{\left(4 \pi \sigma_{i}^{2}\right)^{3 / 2}}{1+2 \mu_{i} T \sigma_{i}^{2}}\right|_{\text {light-nuclei }}
$$

This experimental fact suggests that the coalescence of light nuclei or bound states composed of hadrons will coalesce at different time such that their average phase space that a nucleon occupies is similar to that from statistical model. This being said, we will here just assume that all the molecular configurations are formed at a kinetic freezeout temperature. Then, one finds that the yields of all the molecular configurations are similar to that predicted from statistical model.

\subsection{Multiquark production}

In contrast to the yields of molecular configurations, as has been explicitly shown within a detailed coalescence model calculation, the production of multiquark hadrons are indeed suppressed as the overlap into a compact multiquark configurations are suppressed[8]. The results are shown for candidates of crypto-exotic states in Fig. 2. The study of the yields from both statistical model and 


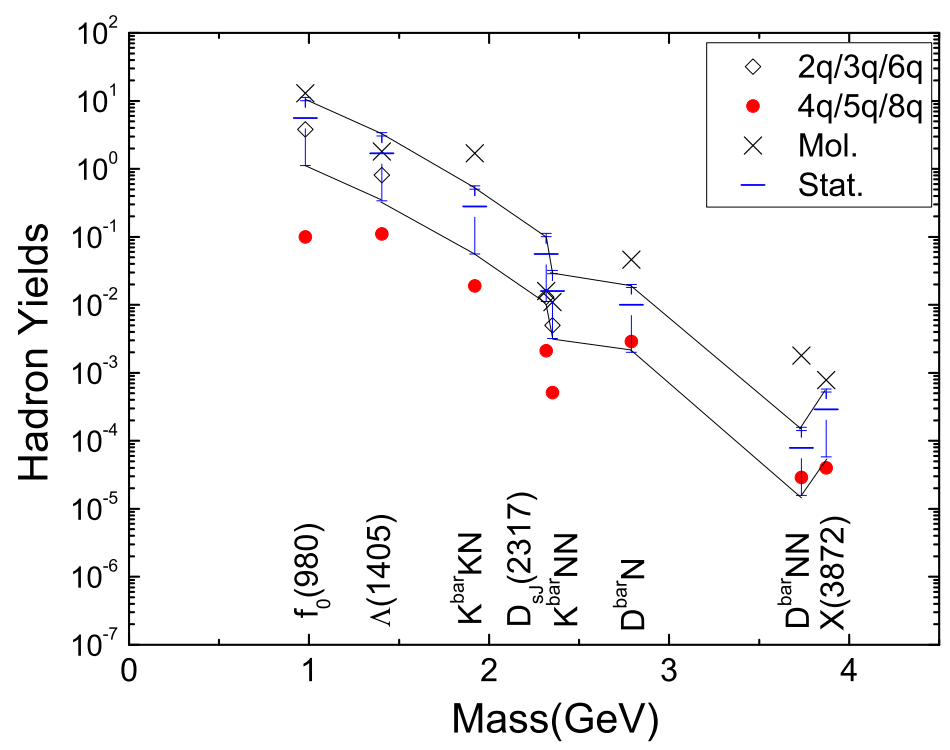

Figure 2. Yields of candidates for multiquark configuration. The yields are suppressed when their structure is of multiquark configurations .

coalescence model shows that all the normal hadron yields from the coalescence model lies in the range between 0.2 and 2 times those estimated from the statistical model. The band in the figure is composed of the two lines obtained by taking 0.2 and 2 times the statistical model prediction for the lower and upper line respectively, implying that hadrons of those yields inside the band must be in normal hadron states. As can be seen from the figure, the yields for most of the candidates are suppressed (below the band) by an order of magnitude to its statistical model prediction when their structure is assumed to be of multiquark nature $(4 q / 5 q / 8 q)$. The only experimental information available are for the $f_{0}$ state measured through its electro magnetic decay. The result lies within the bounds of the statistical model prediction and thus excludes the possibility that its structure is a purely tetraquark state [8].

\section{Heavy exotics from heavy ion collisions}

\subsection{Tetraquark state}

Neither the task of identifying crypto-exotic states nor the attempts to find a flavor exotic state were successful so far. In a series of papers [20,21], we have emphasized that one should search for exotic states with heavy quarks. The reason is simple, the only way one can accommodate many quarks is to minimize the color spin interaction between quarks. Effectively, the strength between two quarks with spin $\vec{s}_{1,2}$ and mass $m_{1,2}$ can be written as

$$
C_{M, B} \vec{s}_{1} \cdot \vec{s}_{2} \frac{1}{m_{1} m_{2}}
$$

where $C_{M}$ and $C_{B}$ are for quark antiquark and quark quark pair respectively. The most attractive channel for quark antiquark is in the color singlet channel and for quark-quark the color antitriplet flavor 
antisymmetric channel. From fitting the meson and baryon mass splitting, it is known phenomenologically that $C_{M} \approx 3 C_{B}$; a fact that is by itself a theoretical challenge to explain. This suggests that for most tetraquark states, the most attractive configuration will inevitably have a large quark antiquark component in the color singlet channel, which will dominantly decay into two mesonic states. On the other hand when the two antiquarks are heavy, the diquark configuration can remain as a dominant and stable configuration. A state that is characterized by such configuration is the $T_{c c}$ state [22].

An excellent place to search for this state is from Belle. The X(3904) was found among the $J / \psi D \bar{D}$ recoil [23]; as two $c \bar{c}$ are produced in these reactions, it could be searched at these reactions. One way of reducing the background is to veto any event involving the strange quark as such event with two $c$ quarks has most likely come from the $b$ decay.

The exact decay mode depends on its exact mass. $T_{c c}$ is most likely a stable state, since its mass is below the threshold of $D^{*} D$. To be more general, one can consider nevertheless both cases where the mass of $T_{c c}$ is above or below the threshold, and discuss in each case possible decay modes that can be realistically detected in experiments with good performance. For the $T_{c c}$ above the threshold of $D^{*} D$, it can decay to $D^{*-} \bar{D}^{0}$ via a strong process. For the $T_{c c}$ below the threshold of $D^{*} D$ and above $D D \pi$, the decay channel to $D^{*-} \bar{D}^{0}$ is energetically forbidden, but the $D^{*-}$ component in $T_{c c}$ can decay through a strong process, leading to the final decay mode $\bar{D}^{0} \bar{D}^{0} \pi^{-}$. On the other hand, when $T_{c c}$ is below the threshold of $D D \pi$, the decay channel of $D^{*-}$ is closed and only the weak decay of $\bar{D}^{0}$ component in $T_{c c}$ is allowed via $\bar{D}^{0} \rightarrow K^{+} \pi^{-}$or $K^{+} \pi^{+} \pi^{-} \pi^{-}$. Therefore, $T_{c c}$ would be detected by the decay modes $D^{*-} K^{+} \pi^{-}$and $D^{*-} K^{+} \pi^{+} \pi^{-} \pi^{-}$. The last two decay patterns would most likely happen since the binding energy of $T_{c c}$ is estimated to be about $80 \mathrm{MeV}$ as shown previously, which is sufficiently larger than the mass difference (about $6 \mathrm{MeV}$ ) between $D^{*-}$ and $\bar{D}^{0} \pi^{-}$. Below the threshold of $D D \pi$, it may be also interesting to see the decay of $D^{*-}$ component in $T_{c c}$. Considering that $D^{*-}$ component contains a quantum number of $\bar{D}^{0} \pi^{-}$, and $\bar{D}^{0}$ decays into $K^{+} \pi^{-}$and $K^{+} \pi^{+} \pi^{-} \pi^{-}$, we may observe the $\bar{D}^{0} K^{+} \pi^{+} \pi^{-}$and $\bar{D}^{0} K^{+} \pi^{+} \pi^{+} \pi^{-} \pi^{-}$decays.

Among the weak decays below the threshold of $D D \pi$, the decay of the $\bar{D}^{0}$ component in $T_{c c}$ can be distinguished from that of the $D^{*-}$ component. The former has the correlations $\left(K^{+} \pi^{-}\right)$ $\left(K^{+} \pi^{-}\right) \pi^{-}$and $\left(K^{+} \pi^{+} \pi^{+} \pi^{-}\right)\left(K^{+} \pi^{-}\right) \pi^{-}$, and the latter has the correlations $\left(K^{+} \pi^{-}\right)\left(K^{+} \pi^{+} \pi^{-}\right)$and $\left(K^{+} \pi^{-}\right)$ $\left(K^{+} \pi^{+} \pi^{-} \pi^{-} \pi^{-}\right)$, where brackets denote correlated particles. However, the $\bar{D}^{0} \bar{D}^{0} \pi^{-}$state, which would appear in $T_{c c}$ in the latter process, contains six quarks, hence further analysis is needed to discuss its stability.

\subsection{Production in heavy ion collisions}

The number of heavy tetraquark mesons produced from the quark-gluon plasma formed in relativistic heavy ion collisions can be estimated in the coalescence model [24], which has been shown to describe very well the pion and proton transverse momentum spectra at intermediate momenta $[15,25]$ as well as at low momenta if resonances are included [14], and the yield and transverse momentum spectra of phi meson and Omega baryon [26] as well as the charmed meson [27]. We employ the formula that was previously used to calculate the yields of tetraquark $D_{s J}(2317)$ meson [17] and pentaquark $\Theta^{+}$baryon [28] at RHIC to study $T_{c c}$ production in central $\mathrm{Au}+\mathrm{Au}$ collisions at RHIC and $\mathrm{Pb}+\mathrm{Pb}$ collisions at LHC. Assuming Bjorken correlation $y=\eta$ between the space-time rapidity $\eta$ and the momentum-energy rapidity $y$ and neglecting the transverse flow as well as using the nonrelativistic approximation, we obtain the following expression for the number of $T_{c c}$ produced from quark coalescence:

$$
N_{T_{c c}} \simeq \frac{1}{432} \frac{N_{\bar{c}} N_{\bar{c}} N_{u} N_{d}}{2} \prod_{i=1}^{3} \frac{\left(4 \pi \sigma_{i}^{2}\right)^{3 / 2}}{V_{C}\left(1+2 \mu_{i} T_{C} \sigma_{i}^{2}\right)}
$$


In the above, $g_{T_{c c}}=3 \times 1 / 3^{4} \times 1 / 2^{4}=1 / 432$ is the color-spin-isospin factor for the four quarks to form a hadron of the quantum number of the tetraquark meson. $T_{C}=170 \mathrm{MeV}$ is the critical temperature and $V_{C}$ is the fireball volume at hadronization, which is about $1,000 \mathrm{fm}^{3}$ in central $\mathrm{Au}+\mathrm{Au}$ collisions at $s_{N N}^{1 / 2}=200 \mathrm{GeV}$ [17] and about $2,700 \mathrm{fm}^{3}$ in central $\mathrm{Pb}+\mathrm{Pb}$ collisions at $s_{N N}^{1 / 2}=5.5 \mathrm{TeV}$ [30]. The quark numbers at hadronization are denoted by $N_{u}$ and $N_{d}$ for light quarks and $N_{c}$ and $N_{\bar{c}}$ for heavy quarks. Their values are taken to be $N_{u}=N_{d}=245$ [17] and 662 [30] as well as $N_{c}=N_{\bar{c}}=3$ and 20 in central RHIC and LHC collisions, respectively, all in one unit of midrapidity. The charm quark numbers are based on initial hard scattering of nucleons in the colliding nuclei $[17,30]$. The width parameter $\sigma_{i}$ in the Wigner function is related to the oscillator frequency $\omega$ by $\sigma_{i}=1 / \sqrt{\mu_{i} \omega}$ with the reduced masses $\mu_{i}$ defined in Eq. (9) of Ref. [17].

Because of the larger abundance of charm quarks at LHC than at RHIC, the number of $T_{c c}$ produced at LHC is more than an order of magnitude larger than that produced at RHIC. For the oscillator frequency $\omega=0.3 \mathrm{GeV}$, determined from the size $\left\langle r_{D_{s}}^{2}\right\rangle_{\mathrm{ch}} \approx 0.124 \mathrm{fm}^{2}$ of the $D_{s}^{+}(c \bar{s})$ meson based on the light-front quark model [29], the number of $T_{c c}$ produced at RHIC and LHC is about $5.5 \times 10^{-6}$ and $9.0 \times 10^{-5}$, respectively.

It is of interest to compare the predicted number of $T_{c c}$ mesons from the coalescence model with that from the statistical model. In this model the number of $T_{c c}$ mesons produced at hadronization is given by [17]:

$$
N_{T_{c c}}^{\text {stat }} \approx \frac{V_{H} \gamma_{C}^{2}}{(2 \pi)^{2}} \int d m_{T} m_{T}^{2} e^{-\frac{\bar{\gamma}_{H} m_{T}}{T_{H}}} I_{0}\left(\frac{\bar{\gamma}_{H} \bar{\beta}_{H} p_{T}}{T_{C}}\right),
$$

where $V_{H}$ and $\bar{\beta}_{H}$ are the volume and radial flow velocity of formed hadronic matter, and $\gamma_{C}$ is the fugacity parameter for ensuring that the number of charmed hadrons produced statistically at hadronization is same as the number of charm quarks in the quark-gluon plasma. With $V_{H} \approx 1,908 \mathrm{fm}^{3}$, $T_{H}=175 \mathrm{MeV}, \bar{\beta}_{H}=0.3 c$, and the charm fugacity $\gamma_{C} \approx 8.4$ [17], we obtain $N_{T_{c c}} \sim 7.5 \times 10^{-4}$ in central $\mathrm{Au}+\mathrm{Au}$ collisions at RHIC. The yield of $T_{c c}$ increases to $8.6 \times 10^{-3}$ in central $\mathrm{Pb}+\mathrm{Pb}$ collisions at LHC where we have used $V_{H} \approx 5,220 \mathrm{fm}^{3}, T_{H}=175 \mathrm{MeV}, \bar{\beta}_{H}=0.47 c$, and the charm fugacity $\gamma_{C} \approx 16.3$ [30]. Compared to those from the coalescence model, predictions from the statistical model are almost two orders of magnitude larger.

\section{Summary}

We have argued that the multiquark states can be identified by measuring their suppressed yields in heavy ion collision. We have also argued that the multiquark states will most likely be found in the heavy sector and the such states could be searched for at Belle and at LHC.

\section{Acknowledgements}

This work was supported by the Korean Research Foundation under BK21 plus program and Grant Nos. KRF-2011-0020333 and KRF-2011-0030621. We thank members of ExHIC collaboration for the joint work.

\section{References}

[1] B. Aubert et al. [BABAR Collaboration]: Observation of a narrow meson decaying to $D_{s}^{+} \pi^{0}$ at a mass of 2.32-GeV/c². Phys. Rev. Lett. 90, 242001 (2003). 
[2] S. K. Choi et al. [Belle Collaboration]: Observation of a new narrow charmonium state in exclusive $B^{+} \rightarrow K^{+} \pi^{+} \pi^{-} J / \psi$ decays. Phys. Rev. Lett. 91, 262001 (2003).

[3] For a recent review see M. Nielsen, F. S. Navarra and S. H. Lee, Phys. Rept. 497, 41 (2010).

[4] R. Aaij et al. [LHCb Collaboration], Phys. Rev. Lett. 110, 222001 (2013) [arXiv:1302.6269 [hepex]].

[5] R. Aaij et al. [LHCb Collaboration], Phys. Rev. Lett. 112, no. 22, 222002 (2014) [arXiv:1404.1903 [hep-ex]].

[6] R. L. Jaffe, Phys. Rev. D 15, 267 (1977); 281 (1977).

[7] R. L. Jaffe, Phys. Rev. Lett. 38, 195 (1977) [Erratum-ibid. 38, 617 (1977)].

[8] S. Cho et al. [ ExHIC Collaboration ], Phys. Rev. Lett. 106, 212001 (2011).

[9] S. Cho et al. [ExHIC Collaboration], Phys. Rev. C 84, 064910 (2011) [arXiv:1107.1302 [nuclth]].

[10] K. Morita, S. H. Lee, Phys. Rev. C77, 064904 (2008).

[11] S. H. Lee, Phys. Rev. D40, 2484 (1989).

[12] G. E. Brown, K. Kubodera, M. Rho, Phys. Lett. B192, 273-278 (1987).

[13] P. Braun-Munzinger, K. Redlich, J. Stachel, In *Hwa, R.C. (ed.) et al.: Quark gluon plasma* 491-599. [nucl-th/0304013].

[14] V. Greco, C. M. Ko and P. Levai, Phys. Rev. Lett. 90, 202302 (2003); Phys. Rev. C 68, 034904 (2003).

[15] R. J. Fries et al., Phys. Rev. Lett. 90, 202303 (2003); Phys. Rev. C 68, 044902 (2003).

[16] Y. Kanada-En'yo and B. Muller, Phys. Rev. C 74, 061901 (2006).

[17] L. W. Chen C. M. Ko, W. Liu and M. Nielsen, Phys. Rev. C 76, 014906 (2007).

[18] H. Agakishiev et al. [STAR Collaboration], Nature 473, 353 (2011) [Erratum-ibid. 475, 412 (2011)] [arXiv:1103.3312 [nucl-ex]].

[19] P. J. Siemens and J. I. Kapusta, Phys. Rev. Lett. 43, 1486 (1979).

[20] S. H. Lee et al., Eur. Phys. J. C 54, 259 (2008).

[21] S. H. Lee and S. Yasui, Eur. Phys. J. C 64, 283 (2009).

[22] S. Zouzou, B. Silvestre-Brac, C. Gignoux and J. M. Richard, Z. Phys. C 30, 457 (1986).

[23] K. Abe et al. [Belle Collaboration] Phys. Rev. Lett. 98, 082001 (2007).

[24] R. Mattiello, A. Jahns, H. Sörge, H. Stöcker, and W. Greiner, Phys. Rev. Lett. 74, 2180 (1995); R. Mattiello,

H. Sörge, H. Stöcker, and W. Greiner, Phys. Rev. C 55, 1443 (1997).

[25] R. C. Hwa and C. B. Yang, Phys. Rev. C 67, 034902 (2003).

[26] L. W. Chen and C. M. Ko, Phys. Rev. C 73, 044903 (2005).

[27] V. Greco, C. M. Ko, and R. Rapp, Phys. Lett. B 595, 202 (2004).

[28] L. W. Chen, V. Greco, C. M. Ko, S. H. Lee, and W. Liu, Phys. Lett. B 601, 34 (2004).

[29] C. W. Hwang, Eur. Phys. J. C 23, 585 (2002).

[30] B. -W. Zhang, C. -M. Ko, W. Liu, Phys. Rev. C77, 024901 (2008). 